\title{
Relative Timing of Isolated Potentials During Postinfarction Ventricular Tachycardia and Sinus Rhythm
}

\author{
Frank Bogun, ${ }^{1}$ Joseph E. Marine, ${ }^{1}$ Stefan $H$. \\ Hohnloser, ${ }^{2}$ Hakan Oral, ${ }^{3}$ Frank Pelosi, ${ }^{3}$ \\ and Fred Morady ${ }^{3}$ \\ ${ }^{1}$ Henry Ford Hospital, Detroit, Michigan, USA; ${ }^{2} J$. W. Goethe \\ University, Division of Cardiology, Frankfurt, Germany; \\ ${ }^{3}$ University of Michigan Medical Center, Ann Arbor, MI, USA
}

\begin{abstract}
Background: In postinfarction patients, isolated potentials separated by an isoelectric segment from the ventricular electrogram indicate areas of block. Isolated potentials can be recorded during both sinus rhythm and ventricular tachycardia (VT). In an attempt to differentiate bystander pathways from critical sites within a reentry circuit, we compared the relative timing of isolated potentials during VT and sinus rhythm.
\end{abstract}

Methods: In 19 patients (mean age $68 \pm 6$ years) with postinfarction VT who were referred for VT ablation, mapping was performed in the presence and absence of VT. Forty-three sites at which there was concealed entrainment during 35 VT's (mean cycle length $469 \pm 74 \mathrm{~ms}$ ) displayed an isolated potential separated from the main portion of the ventricular electrogram by an isoelectric segment of $\geq \mathbf{3 0}$ msec in the presence and absence of VT. The interval between the ventricular electrogram and the isolated potential was measured during VT and baseline rhythm, and the absolute difference $(\Delta$ IPI) was calculated. The $\Delta$ IPI was significantly greater at effective ablation sites $(119 \pm 69 \mathrm{~ms})$ than at ineffective ablation sites $(30 \pm 28 \mathrm{~ms}, p<0.001)$. The positive predictive value of a $\Delta$ IPI $>85 \mathrm{~ms}$ for an effective ablation site was $100 \%$.

Conclusion: At sites of concealed entrainment, an absolute difference $>85 \mathrm{~ms}$ between the isolated potential intervals during sinus rhythm and VT is highly specific for a critical area of the VT reentry circuit in postinfarction patients.

Key Words. ventricular tachycardia, mapping, postinfarct, isolated potentials

\section{Introduction}

Potentials separated from the main portion of the ventricular electrogram in patients with prior myocardial infarction are indicative of an area of block, may occur during systole or diastole [1, 2], and may be present during both ventricular tachycardia (VT) and sinus rhythm [2]. However, isolated potentials are not specific for a critical component of a VT reentry circuit [3]. If an isolated potential originates from a critical component of the reentry circuit, the interval between the ventricular electrogram and the isolated potential during VT and during sinus rhythm may differ substantially (Fig. 1). On the other hand, if an isolated potential originates in a bystander pathway, the interval between the ventricular electrogram and the isolated potential may be similar during VT and during sinus rhythm (Fig. 2). The purpose of the present study was to test this hypothesis by comparing the relative timing of isolated potentials during VT and sinus rhythm at effective and ineffective ablation sites in post-infarction patients with VT.

\section{Methods}

\section{Patient Characteristics}

The 19 patients included in this study were selected from a consecutive series of 45 patients who underwent left ventricular mapping and radiofrequency catheter ablation of postinfarction VT. The selection criteria for this study were the presence of left ventricular sites at which there was concealed entrainment during VT and at which an isolated potential was recorded during both VT and sinus rhythm. There were 16 men and 3 women, and their mean age was $68 \pm 6$ years. The mean left ventricular ejection fraction was $0.23 \pm 0.09$. Among the 19 patients, there were 43 sites during 35 VT's (mean cycle length $469 \pm 74 \mathrm{~ms}$ ) that fulfilled the entry criteria. The baseline rhythm was sinus rhythm in 16 patients, ventricular pacing in

Address for correspondence: Frank Bogun, MD, Henry Ford Hospital, Division of Cardiology K14, 2799 West Grand Blvd, Detroit, MI 48202-9888, USA. E-mail: bogun@eudoramail.com

Received 12 July 2003; accepted 8 September 2003 


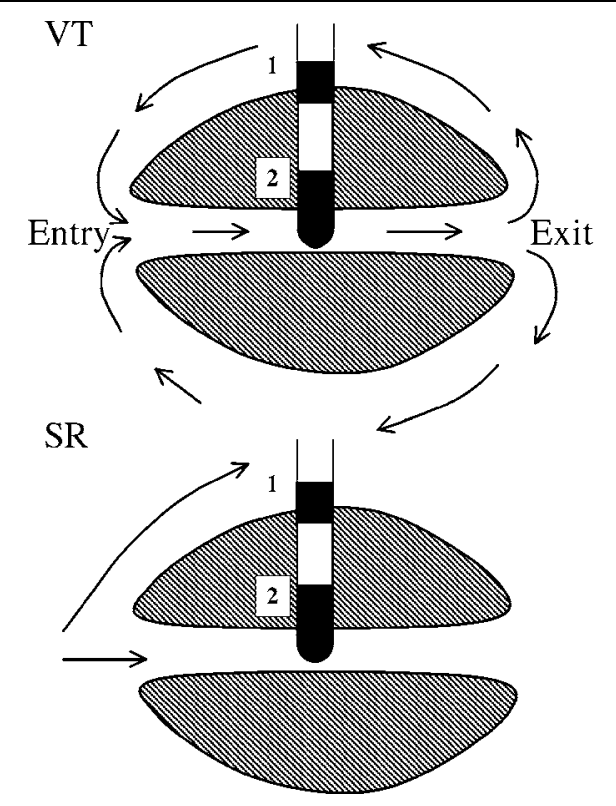

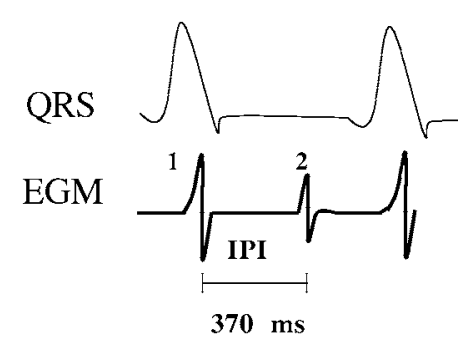

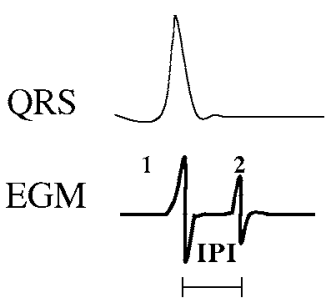

$200 \mathrm{~ms}$

Fig. 1. Schematic depiction of the interval between the ventricular electrogram and isolated potential during VT and during sinus rhythm, when the isolated potential is recorded in a critical portion of a VT reentry circuit. A figure-of-eight model of a reentry circuit is shown, and the cross-hatched areas represent areas of block. The distal pair of electrodes of the mapping catheter is shown. The electrogram recordings (EGM) and the surface QRS complex are displayed on the right. During VT, the mapping catheter records a ventricular electrogram at site \#1 (outside the critical portion of the reentry circuit), and an isolated potential at site \#2 (in the critical common pathway of the reentry circuit). In this example, the interval between the ventricular electrogram and the isolated potential (isolated potential interval, IPI) during VT is $370 \mathrm{~ms}$. During sinus rhythm (SR), because the wave front approaches sites \#1 and \#2 simultaneously, the IPI shortens to $200 \mathrm{~ms}$, resulting in a $\triangle I P I$ of $170 \mathrm{~ms}$.

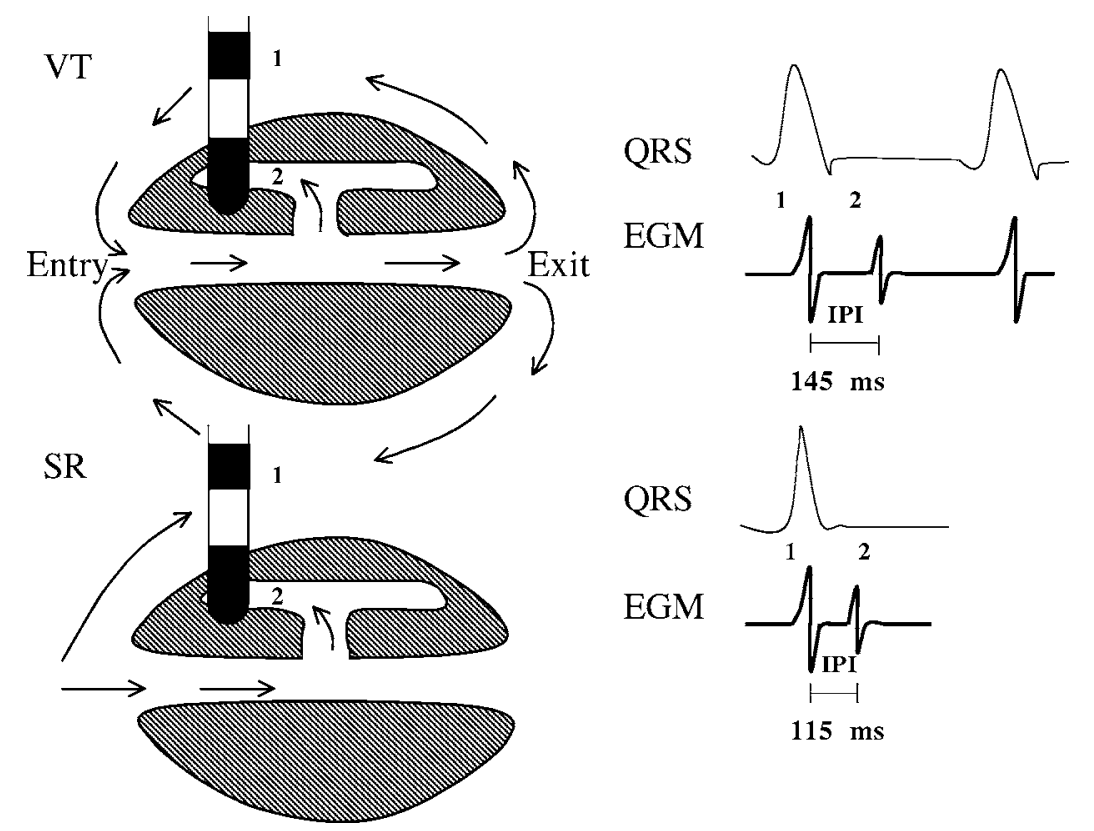

Fig. 2. Schematic depiction of the interval between the ventricular electrogram and isolated potential during VT and during sinus rhythm, when the isolated potential is recorded in a bystander portion of a VT reentry circuit. Format and abbreviations as in Figure 1. During VT, the mapping catheter records a ventricular electrogram at site \#1, outside the critical portion of the reentry circuit, and an isolated potential at site \#2, in a bystander portion of the reentry circuit. The IPI during VT is $145 \mathrm{~ms}$. During sinus rhythm, the IPI shortens minimally to $115 \mathrm{~ms}$, resulting in a $\triangle I P I$ of $30 \mathrm{~ms}$. 
2 patients with underlying high degree AV block, and atrial fibrillation in one patient.

\section{Mapping}

After informed consent was obtained, a $5 \mathrm{Fr}$ quadripolar electrode catheter was inserted into a femoral vein and positioned in the right ventricular apex. Programmed ventricular stimulation was performed with 1-3 extrastimuli to induce VT. A 7 Fr catheter was introduced into a femoral artery and advanced into the left ventricle using a retrograde aortic approach. A 5000-unit bolus of heparin was administered intravenously, followed by 1000 units/hour. A quadripolar electrode catheter with a $4 \mathrm{~mm}$ tip electrode, 2-5-2 interelectrode spacing, and a thermistor embedded in the distal electrode was used for mapping and ablation. Bipolar electrograms were filtered at $50-500 \mathrm{~Hz}$. The intracardiac electrograms and leads V1, I, II and III were displayed on an oscilloscope and recorded and stored digitally (Prucka, Inc, or EP Med Systems). During VT, trains of 10-15 bipolar pacing stimuli were delivered at a cycle length 20-100 ms shorter than the VT cycle length at sites with abnormal electrograms (amplitude $\leq 0.5 \mathrm{mV}$, duration $>60 \mathrm{~ms}$ ). The current strength and pulse width were increased to as much as $10 \mathrm{~mA}$ and $9 \mathrm{~ms}$, respectively, as needed to achieve ventricular capture.

\section{Catheter Ablation}

Radiofrequency energy was applied only at sites at which there was concealed entrainment [3]. Concealed entrainment was defined as entrainment of VT at multiple pacing cycle lengths, without fusion of the QRS complex. Applications of radiofrequency energy were titrated to maintain a target temperature at the electrode-tissue interface of $60^{\circ} \mathrm{C}$, and were delivered during VT. All applications were continued for at least 30 seconds if adequate heating at the electrode-tissue interface was achieved. If VT stopped within 30 seconds, the energy application was continued for a total of 60 seconds. Programmed ventricular stimulation then was repeated. Successful catheter ablation was defined as termination of VT by an application of radiofrequency energy, and the inability to reinduce that VT by programmed stimulation. The mean fluoroscopy time for the procedures was $65 \pm 21$ minutes.

\section{Analysis of Isolated Potentials and Other Mapping Criteria}

A total of 170 sites with concealed entrainment were screened. At 43 of the 170 sites, isolated potentials were present during both ventricular tachycardia and the baseline rhythm. An isolated

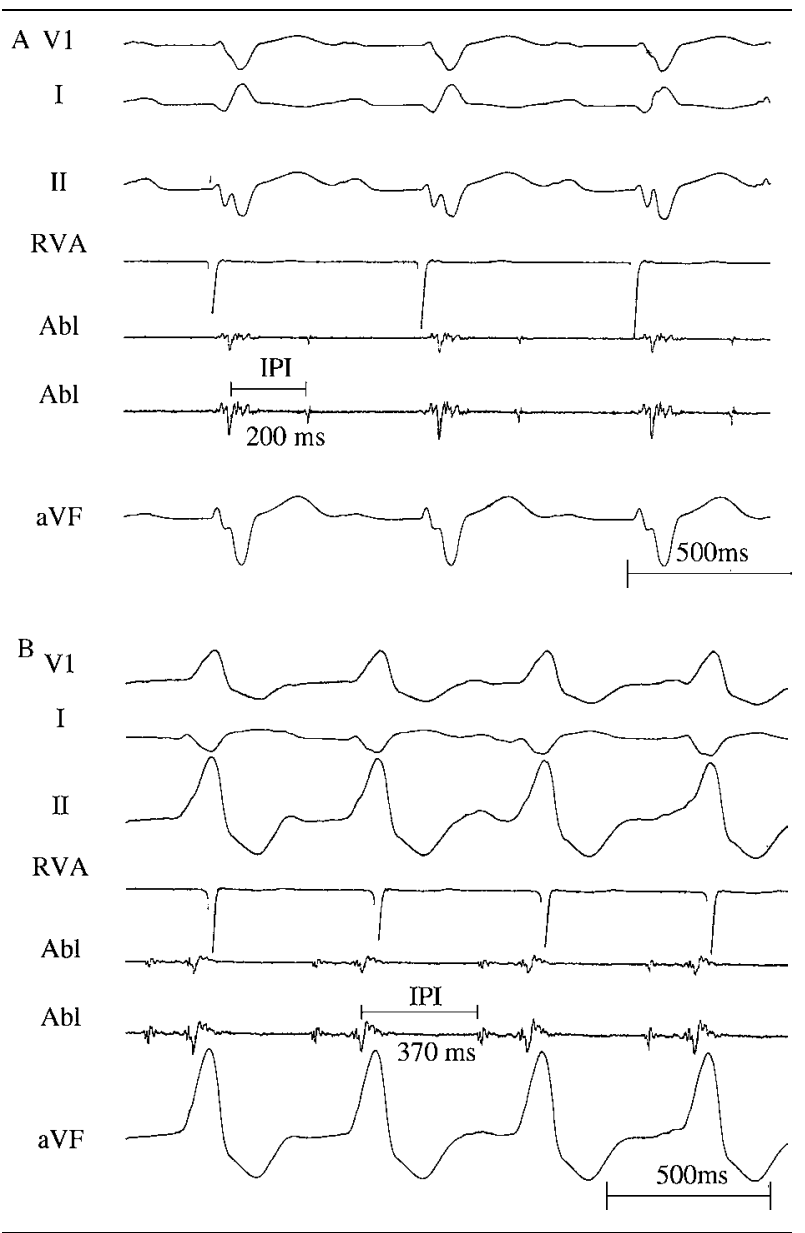

Fig. 3. Isolated potential recorded at an effective ablation site on the anterobasal wall of the left ventricle. Displayed are leads V1, I and II, intracardiac electrograms recorded at the right ventricular apex (RVA) and by the ablation catheter (Abl) at 2 different gain settings (40 and $80 \mathrm{~mm} / \mathrm{mV}$ ), and lead aVF. (A) Isolated potential recorded during sinus rhythm. The isolated potential interval (IPI) is $200 \mathrm{~ms}$. (B) At the same site, the IPI during VT increases to $370 \mathrm{~ms}$. The $\triangle I P I$ is equal to 370 minus $200 \mathrm{~ms}$, or $170 \mathrm{~ms}$.

potential was defined as a potential that was separated from the ventricular electrogram by an isoelectric line of at least $30 \mathrm{~ms}$ (Fig. 3). The interval between the ventricular electrogram and the isolated potential was measured from the points of maximum positive or negative deflection of the electrograms, during the baseline rhythm and during VT (Fig. 3). The $\Delta$ IPI was defined as the absolute difference between the isolated potential interval (IPI) during the baseline rhythm and the IPI during VT. Only sites were selected where distinct electrograms were recorded at the distal and proximal electrodes of the mapping catheter.

In a post-hoc analysis, several other criteria for distinguishing critical from bystander sites in the reentry circuit also were assessed. These 
consisted of the post-pacing interval [4], the stimulus-QRS interval (S-QRS) during concealed entrainment, the S-QRS/VT cycle length ratio, the electrogram-QRS interval, and whether the S-QRS during concealed entrainment was equal to the electrogram-QRS interval.

\section{Statistical Analysis}

Continuous variables are expressed as the mean \pm 1 standard deviation and were compared using Student's t test. Discrete variables were compared using the Fisher exact test or by Chi-square analysis, as appropriate. A $p$ value $<0.05$ was considered significant.

\section{Results}

Among the 43 sites at which an isolated potential was recorded during sinus rhythm and VT, 30 were effective ablation sites and 13 were ineffective ablation sites. Comparisons of the $\Delta$ IPI and other mapping criteria at effective and ineffective ablation sites are presented in Table 1 . The mean $\Delta$ IPI at effective ablation sites was significantly longer than at ineffective ablations sites $(p<0.001)$. A $\Delta$ IPI $>85 \mathrm{~ms}$ was present at $60 \%$ of the effective ablation sites (Fig. 3), compared to $0 \%$ of the ineffective ablation sites (Fig. $4, p<0.001$ ). The S-QRS during concealed entrainment was equal to the electrogram-QRS interval at 73\% of effective ablation sites, compared to $15 \%$ of ineffective ablation sites $(p<0.01)$. There were no significant differences in the post-pacing interval, mean S-QRS, electrogram-QRS interval, or ratio of S-QRS to VT cycle length between effective and ineffective ablation sites.

The individual $\Delta$ IPI intervals at effective and ineffective ablation sites are shown in Figure 5.

Table 1. Comparison of mapping criteria at effective and ineffective VT ablation sites in 19 patients

\begin{tabular}{llll}
\hline Variable & $\begin{array}{l}\text { Effective } \\
\text { sites }(30)\end{array}$ & $\begin{array}{l}\text { Ineffective } \\
\text { sites }(13)\end{array}$ & $P$ \\
\hline$\Delta$ IPI $(\mathrm{ms})$ & $119 \pm 69$ & $30 \pm 28$ & $<0.001$ \\
$\Delta$ IPI $>85 \mathrm{~ms}$ & $18 / 30(60 \%)$ & $0 / 13(0 \%)$ & $<0.001$ \\
S-QRS (ms) & $235 \pm 87$ & $228 \pm 121$ & 0.8 \\
EGM-QRS (ms) & $209 \pm 87$ & $155 \pm 89$ & 0.1 \\
PPI-VTCL (ms) & $104 \pm 132$ & $180 \pm 186$ & 0.2 \\
S-QRS/VT CL $<0.7$ & $23 / 30(77 \%)$ & $7 / 13(54 \%)$ & 0.1 \\
S-QRS = EGM-QRS & $22 / 30(73 \%)$ & $2 / 13(15 \%)$ & $<0.01$ \\
\hline
\end{tabular}

Abbreviations: CL: cycle length; EGM-QRS: electrogram-QRS interval (endocardial activation time); IPI: isolated potential interval; PPIVTCL: VT cycle length subtracted from the postpacing interval; $\triangle \mathrm{IPI}$ : absolute difference between IPI during baseline rhythm and during VT; S-QRS: stimulus-QRS interval; VT: ventricular tachycardia.

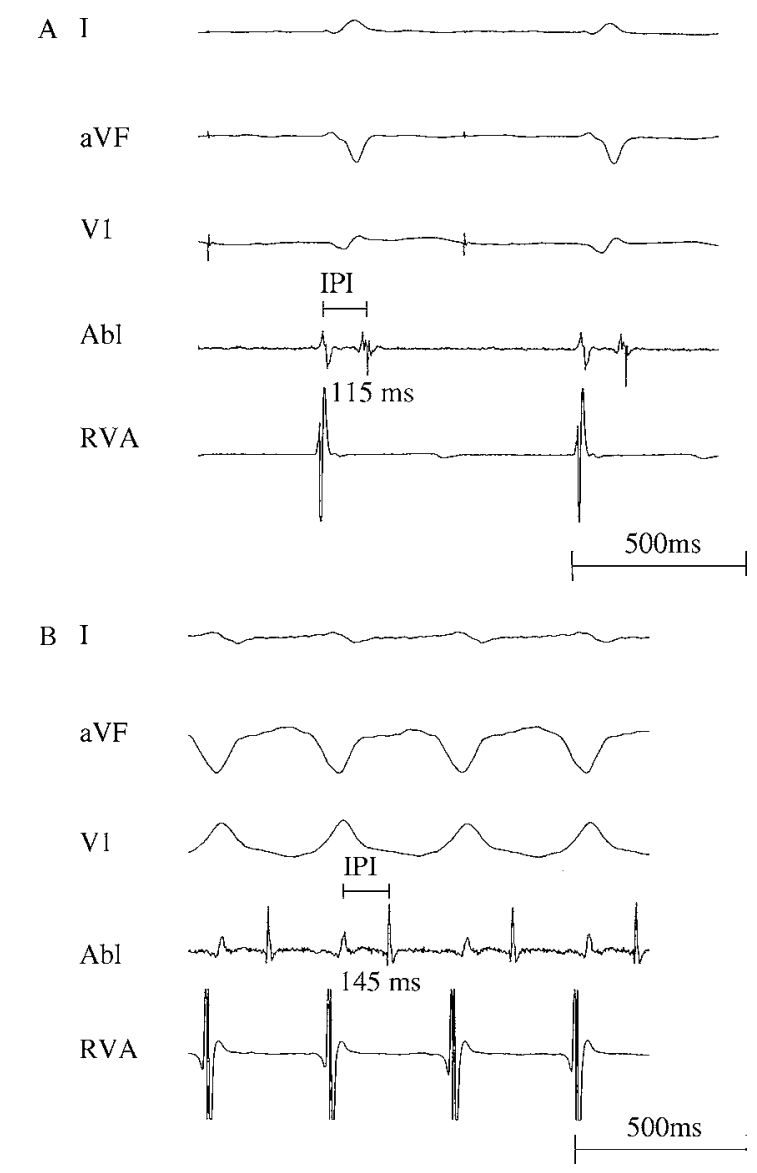

Fig. 4. Isolated potentials recorded at an ineffective ablation site at the mid-anterior wall of the left ventricle. Displayed are leads I, aVF, and V1, and intracardiac electrograms recorded by the ablation catheter $(\mathrm{Abl})$, and at the right ventricular apex (RVA). (A) Isolated potential recorded during atrial pacing. The isolated potential interval (IPI) is $115 \mathrm{~ms}$. (B) At the same recording site, the IPI during VT is $145 \mathrm{~ms}$.

Therefore, the $\triangle I P I$ is equal to 145 minus $115 \mathrm{~ms}$, or $30 \mathrm{~ms}$.

Table 2 compares the sensitivity, specificity, and positive and negative predictive values for an effective ablation site of the $\Delta$ IPI $>85 \mathrm{~ms}$ and of a stimulus-QRS interval equal to the electrogram-QRS interval. The positive predictive value of a $\triangle I P I>85 \mathrm{~ms}$ for an effective ablation site was $100 \%$.

\section{Discussion}

\section{Main Findings}

In this study, the relative timing of isolated potentials during VT and sinus rhythm was compared at sites that demonstrated concealed entrainment of VT. A large (>85 ms) absolute difference in the IPI between VT and sinus rhythm was found to be highly specific for a critical site within the 
Table 2. Sensitivity, specificity, and positive and negative predictive values of mapping criteria for an effective VT ablation site

\begin{tabular}{|c|c|c|c|c|}
\hline Variable & Sensitivity \% & Specificity \% & PPV \% & NPV \% \\
\hline$\Delta \mathrm{IPI}>85 \mathrm{~ms}$ & 60 & 100 & 100 & 52 \\
\hline $\begin{array}{l}\text { S-QRS } \\
\quad=\text { EGM-QRS }\end{array}$ & 73 & 85 & 91 & 58 \\
\hline
\end{tabular}

Abbreviations: PPV: positive predictive value; NPV: negative predictive value; other abbreviations as in Table 1.

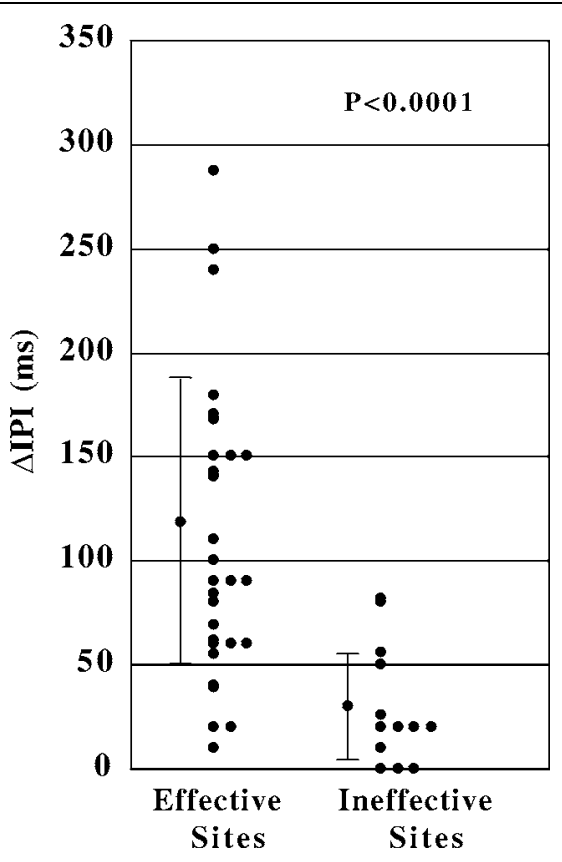

Fig. 5. The $\triangle I P I$ at effective and ineffective ablation sites. The mean \pm standard deviation for each group is also shown. Note that a $\triangle I P I>85 \mathrm{~ms}$ was recorded only at effective ablation sites.

VT reentry circuit. However, when the absolute difference between the isolated potential interval during VT and sinus rhythm was $<85 \mathrm{~ms}$, the probability that the recording site was within a critical component of a reentry circuit was approximately $50 \%$.

\section{Significance of Isolated Potentials}

A prior study demonstrated that isolated potentials during VT often reflect the presence of fixed anatomical barriers within a critical component of a reentry circuit [2]. Consistent with this finding, in an in vitro preparation of human cardiac tissue in which VT was induced, histologic examination of the sites that displayed mid-diastolic potentials during VT demonstrated a surviving myocardial bundle surrounded by fibrous tissue in a zone of infarction $[5,6]$. Furthermore, another study demon- strated that sites that display an isolated potential during VT also may display the isolated potential during sinus rhythm [7]. This suggests that isolated strands of myocardium responsible for a critical component of the reentry circuit can also be detected during sinus rhythm, as demonstrated in this study.

\section{Relative Timing of Isolated Potentials During VT and Sinus Rhythm}

Since isolated potentials may indicate activation of muscle bundles within regions of block, their relative timing during VT and sinus rhythm should depend on whether the recording site is within or outside a critical component of the reentry circuit. As depicted in Figure 1, the components of the reentry circuit are activated in a different sequence during ventricular tachycardia than during sinus rhythm. If the isolated potential is generated within a critical isthmus of the reentry circuit, the IPI interval may be shorter during sinus rhythm than during VT. This would explain why a $>85 \mathrm{~ms}$ difference in $\Delta$ IPI was highly specific for an effective ablation site. On the other hand, if the isolated potential is generated in a bystander region, activation of the bystander area relative to other areas may not differ to a great extent between VT and sinus rhythm (Fig. 2).

At some effective ablation sites, the $\Delta$ IPI was $<85 \mathrm{~ms}$. This may be explained by the position or orientation of the recording electrodes relative to the reentry circuit. For example, as the mapping catheter is positioned nearer to the exit site of the reentry circuit, the $\triangle \mathrm{IPI}$ is accentuated (Fig. 6A). Conversely, the closer the catheter is to the entry site of the reentry circuit, the smaller the $\triangle I P I$ (Fig. 6B). If the ablation catheter is oriented longitudinally relative to the common pathway, the $\triangle$ IPI may be 0 (Fig. $6 \mathrm{C}$ ).

\section{Comparison with Other Criteria}

Among the other mapping criteria that were analyzed in this study, the only criterion other than the $\triangle$ IPI that distinguished effective and ineffective ablation sites was a S-QRS interval during concealed entrainment that was equal to the electrogram-QRS interval [8]. As demonstrated in a prior study, the post-pacing interval was not an effective discriminator, possibly because its accuracy is limited by the assumption that pacing results in a depolarization at the site where subsequent electrograms are recorded [4].

A stimulus-QRS interval/VT cycle length ratio of $<0.7$ did not differentiate effective from ineffective sites in this series. This might be due to a selection bias that excluded sites (such as inner loop sites) at which radiofrequency ablation was 
less likely to be effective. This is in agreement with the findings of Harada et al., who demonstrated that isolated potentials are less frequently observed during sinus rhythm at inner loop sites than at common pathway sites [9].

\section{Limitations}

A limitation of this study is that only sites at which concealed entrainment was present were analyzed. Therefore, the value of the $\Delta$ IPI at sites at which there is not concealed entrainment remains unknown.

Another limitation is that only patients in whom isolated potentials were present during VT and sinus rhythm were included in this study. Because isolated potentials are not present during VT and sinus rhythm in all patients, the overall sensitivity of $\Delta$ IPI for an effective

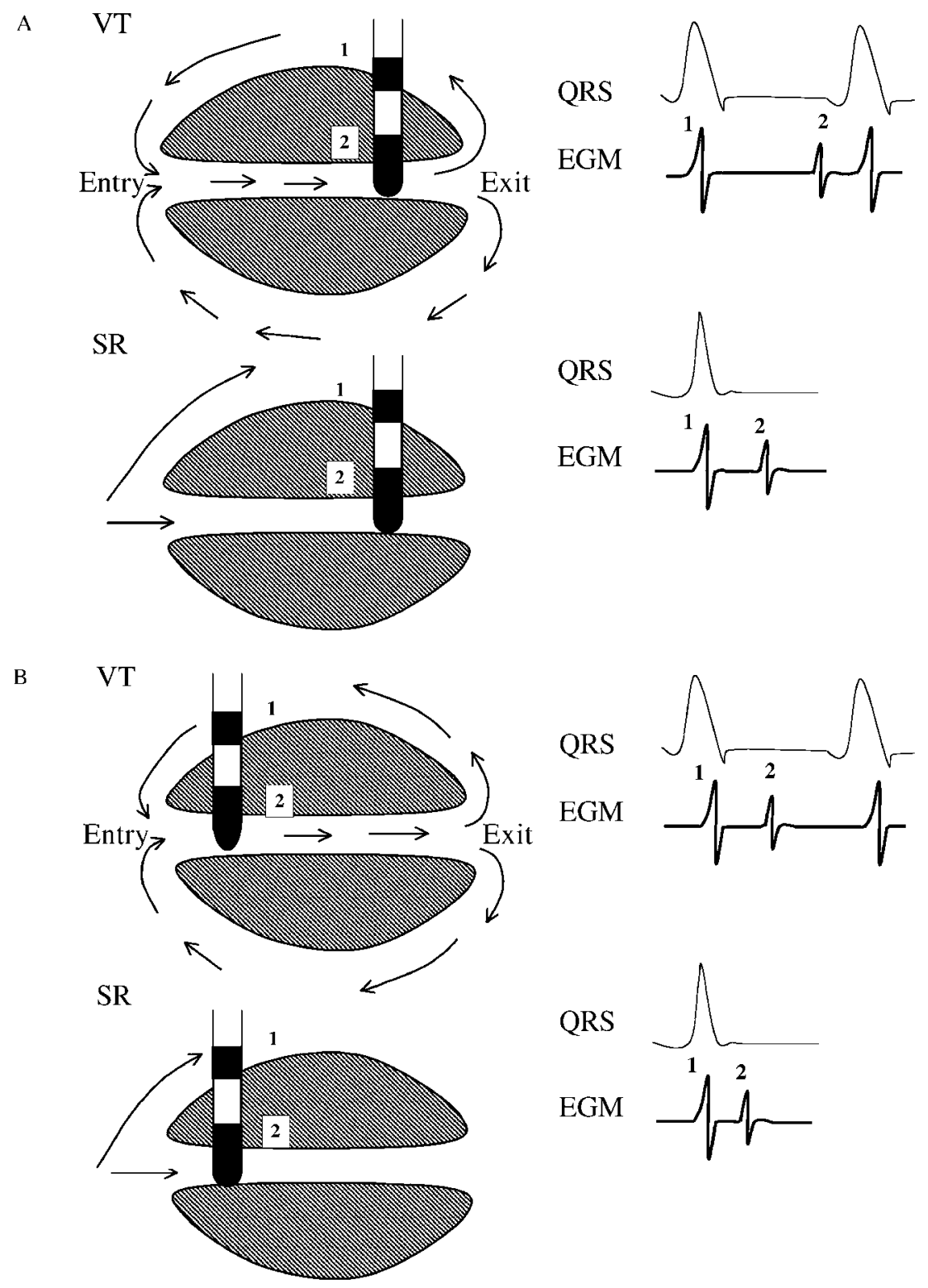

Fig. 6. Examples of how the $\triangle I P I$ at effective ablation sites may be affected by the position and orientation of the electrode catheter to the reentry circuit. (A) If the electrode catheter is positioned closer to the exit site of the reentry circuit, the $\triangle I P I$ is increased. (B) If the electrode catheter is positioned close to the entry site of the reentry circuit, the $\triangle I P I$ becomes smaller. (C) If the electrode catheter is positioned longitudinally over the common pathway, the isoelectric segment between single potentials disappears and the spacing between the individual potentials is identical. Since there is no isoelectric line between individual potentials sites like this were not included in this study. 


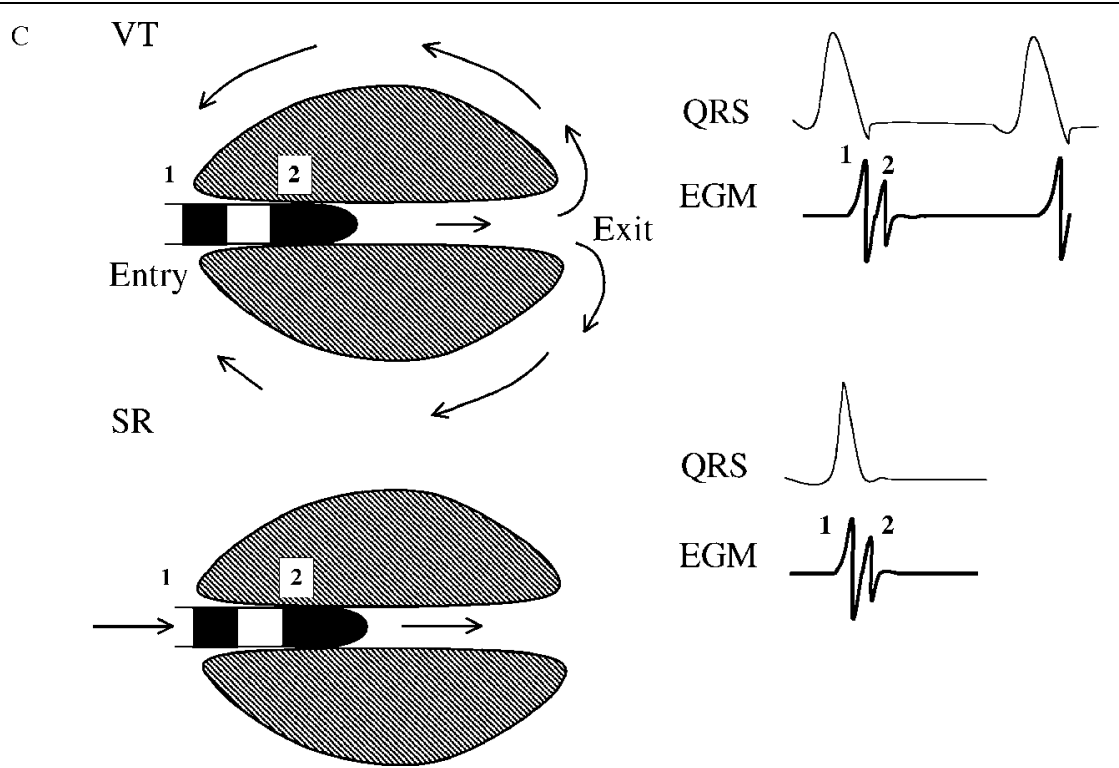

(c)

Fig. 6. (Continued).

ablation site in unselected patients with VT may be lower than determined in this study. The influence of local conduction velocity and dimension of the reentry circuit was not investigated. Conduction velocity would be expected to influence both bystander and critical isthmus sites to the same extend; therefore, the local conduction velocity alone would not explain the higher $\Delta$ IPI that was seen exclusively at critical isthmus sites. With respect to reentry-circuit and bystander pathway dimensions, they certainly impact on the IPI, but this impact would be expected to be similar during VT and sinus rhythm, so that the $\Delta$ IPI would probably not be affected.

In this study, there were only 13 ineffective ablation sites that were used for comparisons with 30 effective ablation sites. Therefore, the findings will require verification with larger sample sizes.

\section{Conclusions}

Because concealed entrainment has a positive predictive value of only $50 \%$ for an effective ablation site, it is useful to have other discriminators of effective and ineffective sites available. This study demonstrates that if an isolated potential is present during VT and sinus rhythm, a $\Delta \mathrm{IPI}>$ 85 has a very high positive predictive value for an effective ablation site.

\section{References}

1. Bogun F, Knight B, Goyal R, Michaud GF, Strickberger SA, Hohnloser SH, Morady F. Discrete systolic potentials during ventricular tachycardia in patients with prior myocardial infarction. J Cardiovasc Electrophysiol 1999;10:364-369.

2. Bogun F, Bahu M, Knight B, Weiss R, Goyal R, Daoud E, Man K, Strickberger S, Morady F. Response to pacing at sites of isolated diastolic potentials during ventricular tachycardia in patients with previous myocardial infarction. J Am Coll Cardiol 1997;30:505-513.

3. Bogun F, Bahu M, Knight B, Weiss R, Paladino W, Harvey M, Goyal R, Daoud E, Man K, Strickberger S, Morady F. Comparison of effective and ineffective target sites that demonstrate concealed entrainment in patients with coronary artery disease undergoing radiofrequency ablation of ventricular tachycardia. Circulation 1997;95:183-190.

4. Bogun F, Knight B, Goyal R, Strickberger SA, Hohnloser $\mathrm{SH}$, Morady F. Clinical value of the postpacing interval for mapping of ventricular tachycardia in patients with prior myocardial infarction. J Cardiovasc Electrophysiol 1999;10:43-51.

5. de Bakker JM, Coronel R, Tasseron S, Wilde AA, Opthof T, Janse MJ, van Capelle FJ, Becker AE, Jambroes G. Ventricular tachycardia in the infarcted, Langendorffperfused human heart: Role of the arrangement of surviving cardiac fibers. J Am Coll Cardiol 1990;15:1594-1607.

6. Gardner PI, Ursell PC, Fenoglio JJ, Jr., Wit AL. Electrophysiologic and anatomic basis for fractionated electrograms recorded from healed myocardial infarcts. Circulation 1985;72:596-611.

7. Bogun F, Bender B, Li Y-G, Groenefeld G, Hohnloser SH, Pelosi F, Knight B, Strickberger SA, Morady F. Analysis during sinus rhythm of critical sites in reentry circuits of 
postinfarction ventricular tachycardia. J Interv Card Electrophysiol 2002;7:95-103.

8. Fontaine G, Tonet F, Grosgogeat Y. Identification of a zone of slow conduction appropriate for VT ablation: Theoretical and practical considerations. PACE 1989;12:262-267.
9. Harada T, Stevenson WG, Kocovic DZ, Friedman PL. Catheter ablation of ventricular tachycardia after myocardial infarction: Relation of endocardial sinus rhythm late potentials to the reentry circuit. J Am Coll Cardiol 1997;30:1015-1023. 